\title{
Die Verfassung Paraguays von 1992 und ihre Vorläufer ${ }^{1}$
}

\author{
Von Anja Schoeller-Schletter
}

\section{Einführung}

"Paraguay ist heute das vielleicht unbekannteste der Länder des lateinamerikanischen Kontinents". Diese Aussage trifft heute noch ebenso zu wie 1963, als Peter A. Schmitt in seiner Untersuchung der Beziehungen zwischen Europa und Paraguay ${ }^{2}$ den Bekanntheitsgrad des Landes so charakterisierte. Was mit Paraguay gemeinhin assoziiert wird, ist weder vielseitig, noch ausgenommen positiv: Paraguay als Sammelpunkt für ehemalige Nazis oder als Zufluchtsort für Steuerhinterzieher; in selteneren Fällen auch als Land des "Jesuitenstaats" im 17. und 18. Jahrhundert.

Im Rahmen des zunehmend bekundeten Interesses Europas, insbesondere Deutschlands, am Ausbau der Handelsbeziehungen zu Südamerika, speziell zu den Ländern des Mercosur ${ }^{3}$, findet Paraguay wenig, jedoch allmählich zunehmende Beachtung. In der Forschung von Geschichts- und Staatswissenschaften wird Paraguay - im Unterschied zu bestimmten anderen Ländern Südamerikas, wie z.B. Chile - bisher so gut wie nicht berücksichtigt. Dabei fanden in den letzten Jahren politische Ereignisse und rechtliche Veränderungen statt $^{4}$, die - vor dem Hintergrund einer sehr eigenwilligen Geschichte, die von langen Abschnitten von Isolation, autoritärer Regierung und politischer Unfreiheit durchzogen ist eine grundlegende Umstrukturierung von Staat und Gesellschaft nach freiheitlich-demo-

Hervorgegangen aus einem Vortrag, den die Verfasserin auf freundliche Anregung von Prof. Dr. Brun-Otto Bryde auf der Jahrestagung des Arbeitskreises Überseeische Verfassungsvergleichung im Juni 1996 in München gehalten hat.

Peter A. Schmitt, Paraguay und Europa. Die diplomatischen Beziehungen unter Carlos Antonio López und Francisco Solano López 1841-1870, Berlin 1963, S. 5.

Z.B. Frankfurter Allgemeine Zeitung vom 16. Sept. 1996, S. 17; 17. Sept. 1996, S. 21; Handelsblatt vom 10.12.1996, S. 12. Zu Aufbau und Struktur der Wirtschaftsintegrationsgemeinschaft MERCOSUR (Mercado Común del Cono Sur) mit den Mitgliedsländern Argentinien, Brasilien, Paraguay, Uruguay: Hartmut Sangmeister, Auf dem Weg in den Weltmarkt: Regionale Wirtschaftsintegration im Cono Sur, in: Detlef Junker / Dieter Nohlen / Hartmut Sangmeister (Hrsg.), Lateinamerika am Ende des 20. Jahrhunderts, München 1994, S. 191 f.

Die politischen Ereignisse 1983-1993 in chronologischer Übersicht: Peter B. Heller, Paraguay. Chronology, in: Albert P. Blaustein / Gisbert H. Flanz (Hrsg.), Constitutions of the Countries of the World, Bd. XIV, New York, Stand 1995; vgl. auch Diego Abente Brun (Hrsg.), Paraguay en transición, Caracas 1993. 
kratischen Prinzipien eingeleitet haben und eine stärkere Einbindung in die internationale Staatengemeinschaft ermöglichen sollen.

Im Februar 1989 wurde General Alfredo Stroessner, seit 1954 Präsident und tragende Persönlichkeit einer insgesamt 34 Jahre dauernden diktatorischen Regierung in Paraguay, durch einen Militärputsch gestürzt. Die Staatsführung übernahm General Andrés Rodríguez, ein ehemaliger Vertrauter Stroessners und Anhänger der reformgewillten Fraktion der traditionellen Regierungspartei der Colorados. In der Folge begann ein politischer Liberalisierungs- und Demokratisierungsprozeß: Oppositionelle Parteien wurden wiederzugelassen, militärische Führungspositionen neu besetzt, die Pressezensur aufgehoben. Nach der offiziellen Wahl von General Rodríguez zum Präsidenten am 1. Mai 1989 und den gleichzeitigen Parlamentswahlen - beides nach den alten Wahlbestimmungen ${ }^{5}$, jedoch unter sehr viel freieren Grundbedingungen - wurde das Wahlrecht reformiert ${ }^{6}$. Im Dezember 1991 fanden Wahlen für eine verfassunggebende Versammlung statt, die unter umfangreicher Beteiligung verschiedener politisch und gesellschaftlich relevanter Gruppen, einschließlich zahlreicher NGOs (non-governmental organisations), eine neue Verfassung ausarbeiten sollte. ${ }^{7}$

Als staatsrechtlich grundlegender Schritt wurde am 20. Juni 1992 durch diese Versammlung eine neue paraguayische Verfassung verabschiedet, welche eine "democracia representativa, participativa y pluralista" als Staats- und Regierungsform festlegte (Art. 1). ${ }^{8}$ Die neue Verfassung proklamiert Demokratie nicht nur als regierungsbestimmendes Prinzip auch die Vorgängerverfassungen von 1940 und 1967 deklarierten die mit ihnen jeweils begründete Staats- und Regierungsform als democracia representativa -, sondern gestaltet das Prinzip im Zusammenspiel mit anderen Strukturentscheidungen in einer Weise aus, die ihre national wie auch international anerkannte Gesamtqualifizierung als demokratische Verfassung rechtfertigen kann. Auf der Grundlage dieser Verfassung wurde im Mai 1993

Estatuto Electoral, Ley 886 vom 21. Dezember 1981, mit kritischen Anmerkungen: Justo José Prieto, El Estatuto Electoral cuestionado, Asunción 1988. Das Gesetz legt u.a. die Mehrheitswahl fest: 2/3 der zu vergebenden Sitze fallen an die Partei mit relativer Mehrheit, 1/3 wird den Wahlergebnissen entsprechend auf die übrigen Parteien verteilt. Zur Entwicklung des Wahlrechts, wenn auch nicht auf dem neusten Stand, M. León-Roesch, Paraguay, in: Handbuch der Wahldaten Lateinamerikas und der Karibik, Opladen 1993, S. 631 ff.

Codigo Electoral von 1990 (Ley No.1/ 90).

Die verschiedenen Verfassungsprojekte sind publiziert in: C.T. Pappalardo Zaldívar, Reforma constitucional. Proyectos y aportes, 2 Bde., Asunción 1992; zur Reformdebatte auch Rafael Dendia / Luis Lezcano Claude, Constitución y Cambio. Aportes para el Debate de la Convención Nacional Constituyente, 2 Bde., Asunción 1991 (CIDSEP); Carlos Mateo Balmelli, Zur gegenwärtigen Verfassungsdiskussion in ausgewählten lateinamerikanischen Ländern: Chile, Paraguay, Argentinien, Frankfurt a.M. 1992, S. 96 ff.

Constitución de la Républica del Paraguay, veröffentlicht in: Las Constituciones de Iberoamerica, hrsg. von Luis Lopez Guerra und Luis Aguiar de Luque, Madrid 1992, S. 737 ff.; in englischer Übersetzung bei Albert P. Blaustein / Gisbert H. Flanz (Hrsg.), a.a.O. 
der Ingenieur Juan Carlos Wasmosy, Kandidat des gemäßigten Flügels der Colorados, zum Präsidenten der Republik Paraguay gewählt - als erster ziviler Regierungschef in fast 50 Jahren; im Parlament erhielten die Oppositionsparteien die Mehrheit.

Mit der Verabschiedung der Verfassung von 1992 und den damit einhergehenden Veränderungen, folgt Paraguay einer allgemeinen staats- und verfassungspolitischen Entwicklungstendenz der 80er und frühen 90er Jahre in Lateinamerika: Der Tendenz zur Ablösung von Militärdiktaturen durch verfassungsrechtlich demokratische Staats- und Regierungsformen mit entsprechend legitimiertem zivilen Staatsoberhaupt. ${ }^{9}$ Zwar gab es in den vorangegangenen Jahrzehnten von Militärregierungen, speziell in den 60er Jahren, Ansätze zur Demokratisierung der Verfassungen; diese beschränkten sich jedoch im Grunde auf die formale Fassade und waren durch den Rückgriff auf die v.a. den Ängsten der USA entsprechende Argumentation, den Kommunismus bei allen geforderten Demokratisierungsmaßnahmen bekämpfen zu müssen, nicht sehr durchgreifend. Vor dem veränderten weltpolitischen Hintergrund der späten 80er und 90er Jahre kamen in verschiedenen Ländern Südamerikas erneut und verstärkt Demokratisierungsbestrebungen zum Tragen, wie beispielsweise in Chile und Paraguay.

Der Reformprozeß in Paraguay ist in vielerlei Hinsicht als Reaktion auf die politische und rechtliche Situation der Stroessner-Zeit zu verstehen, die Verfassung von 1992 auch als Ausdruck des Versuchs, auf die Schwachstellen der Verfassung von 1967 und die in vielerlei Hinsicht im Kontrast zu ihr stehende Verfassungsrealität zu reagieren. Dabei steht die neueste Verfassungsentwicklung zwiespältig vor dem Hintergrund der verfassungsrechtlichen, politischen und gesellschaftlichen Tradition, von dem sie sich einerseits absetzt, in dem sie jedoch andererseits die Bedingungen hat, auf denen sie aufbauen muß. So ist der konkrete Inhalt der Verfassung von 1992 - die Statuierung gerade ganz bestimmter Rechtsinstitute, Organe und Kompetenzverteilungen - Ausdruck des spezifischen Umgangs mit diesem Hintergrund, im Sinne der Bemerkung Richard Thomas, daß jedes staatsrechtliche Institut auch den "im Strom der Geschichte stehenden Versuch der Lösung eines politischen Problems" darstellt ${ }^{10}$. Der Reformprozeß erfolgt als ein "proceso de transición", in dem sich ein "konfliktreicher Wechsel der politischen Kräfte zwischen dem langsam absterbenden autoritären System und dem auflebenden demokratischen Prozeß" vollziehen

9 Vgl. Nikolaus Werz, Demokratie und Regierungsformen in Südamerika, in: VRÜ 20 (1987), S. $139 \mathrm{ff}$., mit weiteren Literaturhinweisen. Zum Wechsel der Regierungsformen, sowie zu den Entwicklungsperspektiven für die 90er Jahre: Dieter Nohlen, Lateinamerika zwischen Diktatur und Demokratie, in: Junker / Nohlen / Sangmeister (Hrsg.), a.a.O., S. 12 ff.

10 Richard Thoma in: ders. und G. Anschütz (Hrsg.), Handbuch des deutschen Staatsrechts, Bd.I, Tübingen 1930, S. 5. 
muß ${ }^{11}$, ohne radikalen Bruch - mittels einer Verfassungsreform unter Einhaltung des in der Verfassung von 1967 vorgeschriebenen Verfahrens, bei Übernahme der vorhandenen Staatsorgane und -institutionen, und ohne derart harter Abrechnung mit der Vergangenheit wie beispielsweise in Chile nach Pinochet.

\section{Verfassungsgeschichtlicher Hintergrund}

Für die Verfassungsgeschichte Paraguays gilt, was die lateinamerikanische Verfassungsgeschichte allgemein kennzeichnet: Sie ist in hohem Grade Rezeptionsgeschichte. Immer wieder ist auf europäische bzw. U.S.-amerikanische Verfassungskonzepte zurückgegriffen worden, wurden Organe und Rechtsinstitute - häufig mehr dem Namen nach als in ideengetreuer Gestalt - vor dem Hintergrund der landeseigenen Gesellschaftsstrukturen in die Regierungsorganisation eingefügt; manche von ihnen machten eine eigenständige Entwicklung durch, bildeten spezielle Charakteristika aus, und formten so eine eigene Verfassungstradition.

\section{Das Regierungsstatut von 1813, die Verfassung von 1844 und die Verfassung von 1870}

Als Beginn der Verfassungsgeschichte Paraguays am Ende der spanischen Kolonialzeit kann das Regierungsstatut von $1813^{12}$ angesehen werden, das ein Konsuln-System begründet. Demgemäß war die Regierungsgewalt von zwei Cónsules de la República del Paraguay gemeinschaftlich auszuüben (Art. 2). Die Zuständigkeitsbefugnis umfaßte alle Bereiche, einschließlich der Rechtsprechung (Art. 12). Vorbild für die mit dem Reglamento de Gobierno 1813 in Paraguay eingeführte Konsulnregierung waren bis zu einem gewissen Grad die französische Republik und das Konsuln-System, welches Napoleon mittels Staatsstreichs 1799 in Frankreich einführte.

Die Verfassung von $1844^{13}$ konstituiert ein Präsidialsystem mit gewaltengegliederter Regierung. Exekutive, Legislative und Judikative werden als drei jeweils eigene Zweige der Regierung verstanden (Titulo I, Art. 1 bis 4). Dem Präsidenten der Republik kam insbesondere durch eine lange Amtszeit (10 Jahre), die geringe Mitwirkungsmöglichkeit des Kon-

11

12

13

Ramiro Barboza, Constitución de la República del Paraguay 1992. Convención Nacional Constituyente, Tomo I (2 Bde.), Asunción 1993 (CIDSEP-AID), Introducción.

Reglamento de Gobiemo de 1813, abgedruckt in C. Pappalardo Zaldívar, Paraguay: Itinerario Constitucional, Asunción 1991 (2. ed.), S. $13 \mathrm{ff}$.

"Ley que establece la administración política de la República del Paraguay, y demás que en ella se contiene", in: C. Pappalardo Zaldívar, a.a.O., S. $67 \mathrm{ff}$. 
gresses - die 200 Abgeordneten traten nur alle fünf Jahre zusammen -, sowie durch eine Fülle weitreichender Befugnisse (Tit. VII, Art. 1 bis 30) eine besonders starke Stellung innerhalb des Regierungssystems zu. Die Befugnisse des Congreso bzw. der Legislatura Nacional umfassen u.a. auch die Wahl des Präsidenten der Republik (Tit. V) und die Errichtung von Gerichtshöfen sowie die Regelung der Verfahren (Tit. III, Art. 8). Die Organisation der Justiz war mit dem 1842 verabschiedeten Estatuto provisorio de la administración de justicia ${ }^{14}$ geregelt worden. Dem Präsidenten ist die ausschließliche richterliche Kompetenz in Sachen Landesverrat und Verschwörung gegen die öffentliche Ordnung vorbehalten (Tit. VII, Art. 18), auch die Ernennung und Absetzung der Richter. Eine Neuerung, die die Verfassung von 1844 (Tit. IX) hinsichtlich der Staatsorganisation mit sich bringt, ist die Einführung des Consejo de Estado ("Staatsrat"), welcher später in den Verfassungen von 1940 (Art. 62 ff.) und 1967 (Art. 188 ff.) wiederauftaucht. Es handelt sich um ein beratendes Organ, dessen Mitglieder vom jeweiligen Präsidenten bestellt werden. ${ }^{15}$ Im Rahmen von allgemeinen Anordnungen am Ende der Verfassung wird u.a. die Gleichheit vor dem Gesetz festgelegt und das Recht auf Anhörung, sowie die Freizügigkeit, die Presse, das Bildungswesen einschränkend geregelt - d.h. von der Erteilung einer Regierungsgenehmigung abhängig gemacht - und der Sklavenhandel verboten (Tit. X).

In der Verfassung von 1844 zeigten sich - hinsichtlich der Aufgliederung der Regierungsgewalt und der Benennung der Rechtsorgane - im Vergleich zu den vorangegengenen regierungskonstituierenden Dokumenten, grundsätzliche Veränderungen in Aufbau und Aussehen. Jedoch hatte sich an der Funktion des Kongresses und der machtpolitischen Stellung des obersten Exekutivorgans grundsätzlich nicht viel geändert: Nach wie vor trat der Kongress in derart großen Abständen zusammen, daß von einer legislativen Funktion i.S.v. Regierungsgestaltung durch Gesetzgebung nur in äußerst geringem Maße gesprochen werden kann; nach wie vor diente der Kongreß als Instanz, die dem Ereignis - sei es die Ratifizierung der formellen Unabhängigkeitserklärung 1842 oder die Konstituierung einer Regierung bzw. deren Bestätigung - eine bestimmte Legitimität dadurch verlieh, daß er als "repräsentatives" Organ zustimmte ${ }^{16}$.

Nach dem s.g. Triple Alliance Krieg, in dem Paraguay in sechs Jahren (1864-1870) drei Viertel seiner Bevölkerung verlor und große Teile seines Territoriums abtreten mußte,

Zum Estatuto Provisorio de Justicia von 1842 und den damit einhergehenden Neuerungen für die Justiz vgl. Juan José Soler, Introducción al Derecho Paraguayo, Madrid 1954, S. 241-244.

Die Einführung des Conse jo de Estado in die Verfassung Paraguays von 1844 - wie auch seine Übernahme in andere lateinamerikanische Verfassungen, z.B. in die von Chile 1823 - läßt sich auf die spanische Verfassung von 1812 zurückführen. Auch im napoleonischen Konsulnsystem von 1799 existierte ein Conseil d'Etat.

16 Vgl. Esteban Caballero / Alejandro Vial (Hrsg.), Poder Legislativo en el Cono Sur, Vol.II, Asunción 1994, S. 71. 
wurde in dem noch von den Siegermächten besetzten Paraguay 1870 eine neue Verfassung verabschiedet. ${ }^{17}$ Ausgerichtet an der argentinischen Verfassung von 1853 entspricht sie damit auch mit einigen, teilweise grundsätzlichen Unterschieden (Katholizismus als Staatsreligion, Eröffnung und außerordentliche Einberufung des Kongresses durch den Staatspräsidenten, etc.) der Verfassung der USA, stellt also im System der Gewaltenteilung und gegenseitigen Kontrolle (checks and balances) ein ausgeglichenes Verhältnis zwischen Exekutive, Legislative und Judikative her. Wie in den Verfassungen der USA und Argentiniens bestand das Legislativorgan aus zwei Kammern, die wegen des unitarischen Charakters des Repräsentationssystems aber mehr oder weniger nach dem gleichen Wahlverfahren gewählt wurden (Art. 43 und 51). Erstmals umfaßte die Verfassung - in Anlehung an die Bill of Rights der USA - auch einen umfangreichen Grundrechtskatalog. Unter der siebzigjährigen Geltungsdauer der Verfassung von 1870, die von geringer demokratischer Regierungserfahrung geprägt ist, wechselten sich unter zahlreichen Präsidenten autoritäre, chaotische und stabilere Zeiten ab. ${ }^{18}$ In die ersten Jahre unter der 1870er Verfassung fällt die Entstehung der beiden traditionellen Parteien Paraguays, der - anfangs von Argentinien unterstützten - Partei der Liberalen und der - nationalkonservativen - Colorado-Partei, von denen letztere die Regierungen 1878-1904 dominierte, erstere die jenigen von 1904-1940.

\section{Die Verfassungen von 1940 und 1967}

Die Verfassung von 1940 ist um vieles stärker von den konkreten gesellschaftlichen Entwicklungen in Paraguay geprägt. Die Auswirkungen der Weltwirtschaftskrise, die instabile liberale Regierung, die sich verbreitende Enttäuschung über die liberale Politik und das nach einem weiteren Krieg - dem von 1932 bis 1935 gegen Bolivien siegreich geführten Chaco-Krieg $^{19}$ - wiederauflebende nationale Bewußtsein, begünstigten die Verbreitung von nationalsozialistischem und faschistischem Gedankengut, ließen soziale Forderungen und den Ruf nach einer starken Regierung laut werden. Es kam zu einem Militärputsch und einer zwischenzeitlichen (18 Monate) Außerkraftsetzung der Verfassung von $1870 .^{20}$ Der 1940 zum Präsidenten gewählte General José Felix Estigarribia gab dem Reformdruck nach und setzte - entgegen seiner Ankündigung, eine verfassunggebende Versammlung einzube-

Constitución de la Républica del Paraguay (1870), publiziert in: C. Pappalardo Zaldívar, Paraguay: Itinerario Constitucional, 1992, S. $81 \mathrm{ff}$.

Zur politischen und wirtschaftlichen Entwicklung der Zeit nach 1870: Encyclopedia of Latin American History and Culture, hrsg. von Barbara A. Tenenbaum, vol. 4, New York 1996, Stichwort Paraguay, S. 295 f.

19

Dazu kürzlich erschienen: Michael Herzig, Der Chaco-Krieg zwischen Bolivien und Paraguay 1932-1935 (Hispano-Amerika Bd. 12), Frankfurt 1996.

20

Dekret vom 19 Februar 1936 und Dekret No. 152 vom 10. März 1936, in: C. Pappalardo Zaldivar, Paraguay: Itinerario Constitucional, 1992, S. 105 f., 107 ff. 
rufen $^{21}$ - per Dekret eine neue Verfassung ein ${ }^{22}$, die zur Bestätigung nachträglich einem Plebiszit - dem zweiten und bisher letzten in der paraguayischen Verfassungsgeschichte ${ }^{23}$ - unterzogen wurde. Mit der neu festgesetzten Staatsordnung verband sich eine Abkehr von der mit der Verfassung von 1870 angestrebten liberal-demokratischen Regierungsform mit einem Gleichgewicht zwischen den Gewalten. Die Verfassung von 1940 war von dem teils korporativ strukturierten "Estado Novo" in Brasilien beeinflußt, der sich wiederum v.a. an Italien und Portugal orientiert hatte ${ }^{24}$. Anders als in der Verfassung von 1870 sah sie - wie 1844 - eine Ein-Kammer-Legislative und einen nicht gewählten Consejo de Estado vor, dessen Mitglieder aus Landwirtschaft, Handel, Industrie, Nationalbank, Militär, Universität und katholischer Kirche rekrutiert wurden, und der so als Vertretung der Interessen dieser Gruppen fungieren sollte. Seine Aufgaben umfaßten die Beratung über Regierungsprojekte und Mitwirkung bei Stellenbesetzungen in Form der Zutimmung, u.a. auch bei der Ernennung der Richter der Corte Suprema (Art. 62-66). Die Exekutive - die auch in der Gliederung der Verfassung ihren Platz vor der Legislative einnahm - wurde durch umfassende Kompetenzerweiterungen gestärkt (Auflösung des Kongresses, Erlaß von Dekreten mit Gesetzeskraft während des Rezesses, Erklärung des Ausnahmezustands, etc.). Der Gerichtsbarkeit wurde vor allem durch das Besetzungsverfahren die Unabhängigkeit genommen (Art. 84). Bei den Grundrechten gab es Einschränkungen; Normen mit sozialen Inhalten wurden eingefügt und weitgehende staatliche Eingriffe in das Wirtschaftsleben ermöglicht. Nach dem Tod Estigarribias noch im selben Jahr folgten Jahre mit verschiedenen Colorado-Militär-Diktaturen. Die Liberale Partei war seit 1942 verboten, die Sympathien für die Nazionalsozialisten hatten sich verbreitet. Im Anschluß an den Sieg der Alliierten in Europa wurden unter dem Druck der USA die Oppositionsparteien wiederzugelassen, auch die Kommunistische Partei. Nach einem Bürgerkrieg und erneuten Colorado-Militär-Regierungen übernahm im Mai 1954 General Alfredo Stroessner durch einen Militärputsch die Regierung, die er - nachdem er sich zum Präsidenten hatte wählen lassen - unter Geltung der Verfassung von 1940 für die folgenden 13 Jahre ausübte.

Im Jahre 1967 wurde eine verfassunggebende Versammlung einberufen, mit dem vorrangigen Ziel, weitere Amtsperioden Stroessners zu ermöglichen, denn die bis dahin gültige Verfassung begrenzte das Präsidentenamt auf fünf Jahre und einmalige Wiederwahl, also eine Amtszeit von 10 Jahren, die Stroessner schon hinter sich hatte. Unter Beteiligung der

Proclama del General José Felix Estigarribia, 18. Februar 1940, in: C. Pappalardo Zaldívar a.a.O., S. $110 \mathrm{ff}$.

Verfassung von 1940 (Decreto Ley No. 2242 vom 10 Juli 1940), in : C. Pappalardo Zaldívar, a.a.O., S. $115 \mathrm{ff}$.

Vor der Ratifizierung des Friedensvertrags vom 21. Juli 1938 zwischen Bolivien und Paraguay mit der Grenzfestlegung nach dem Chaco-Krieg war schon einmal ein Plebiszit (10. August 1938) durchgeführt worden. 
Oppositionsparteien, deren anfängliche Hoffnungen auf den Beginn eines Reformprozesses im Ergebnis enttäuscht wurden, verabschiedete die Kommission eine neue Verfassung, die entscheidende Teile der Verfassung von 1940 und die Kompetenzenfülle der Exekutive beibehielt. ${ }^{25}$ Die Legislative ist durch die Wiedereinführung des Senats zum Zwei-Kammer-System geworden, der Conse jo de Estado blieb als rein beratendes Organ der Exekutive erhalten (Art. 188-192). Die Zustimmung zur Ernennung der Richter der Corte Suprema, die zuvor zu den Zuständigkeiten des Consejo de Estado gehörte, ging auf den vom Volk gewählten Senat über (Art. 151). Die Bestimmung zur Amtszeit des Präsidenten wurde übernommen, die Zeitberechnung begann jedoch erst 1968, so daß Stroessner für zwei weitere Amtszeiten gewählt werden konnte. Abgesehen von einer Verfassungsänderung im Jahre 1977, mit der die unbegrenzte Wiederwahlmöglichkeit des Präsidenten festgelegt wurde, blieb die Verfassung in unveränderter Form bis zu ihrer Ablösung im Jahr 1992 in Kraft.

\section{Verfassungstradition, Verfassungsrealität und die Verfassung von 1992 - Defizite, Probleme und Lösungsansätze}

Versucht man, die Verfassungstradition Paraguays auf einige grundlegende Merkmale festzulegen, können als Konstanten der in den Verfassungen verankerten Strukturen und Staatsorgane folgende gelten: zum Einen ein Präsidialsystem mit starkem Staatsoberhaupt mit Kennzeichen einer christlich-hierarchisch-patriarchalischen Tradition, welche auch als Grundstruktur viele Gesellschaftsbereiche prägt; in diesem Zusammenhang steht die Festlegung des Römisch-Katholischen als Staatsreligion. Verstärkt dadurch, daß in der politischen Praxis das Präsidentenamt häufig über lange Zeit an eine Persönlichkeit gebunden war, werden verfassungsrechtliche Einschränkungen seiner Macht so gut wie unwirksam. Die Ausnahmezustandregelung, - mit Vorläufer in der Verfassung von 1844, und als praktisch uneingeschränkte Befugnis des Präsidenten in den Verfassungen von 1940 und 1967 - hat sich als besonders effektives Instrument erwiesen. Die enge Verbindung zwischen Exekutive und Militär zieht sich durch die gesamte Entwicklungsgeschichte. Daneben steht ein verfassungsrechtlich schwaches Parlament, dem faktisch vornehmlich Legitimationsfunktion zukommt. Als besondere Kompetenzeinschränkung des Parlaments hat sich die Befugnis des Präsidenten zur Parlamentsauflösung sowie zum Erlaß von Dekret-Gesetzen in Zeiten, in denen der Kongreß nicht zusammentritt, herausgebildet. In Aufgabenkonkurrenz zum Senat kann der Consejo de Estado als Teil der Verfassungstradition gesehen werden, welcher sich im Unterschied zu einem Ministerrat dadurch auszeichnet, daß er sich

Verfassung von 1967, in: ders., S. 134 ff.; mit Index und Konkordanzen Justo J. Prieto, La Constitución Paraguaya Concordada, Asunción 1981; Sitzungsprotokolle veröffentlicht als: Convención Nacional Constituyente de la República del Paraguay, Diario de Sesiones 23 Mayo al 25 de Augosto 1967, Tomo I-V, Asunción 1974-1975. 
nicht aus Leitern von Fachressorts zusammensetzt, sondern aus Vertretern bestimmter, als wichtig erachteter Gesellschaftsbereiche, die der Exekutive beratend zur Seite gestellt sind. Die Judikative in Form des Obersten Gerichtshofs hat als Organ zur Gewährleistung der verfassungsmäßig festgelegten Rechte keine Tradition in Paraguay.

Mit der Verabschiedung der Verfassung von 1992 sollten die zuvor bestehenden verfassungsrechtlichen - an den Vorstellungen von Demokratie und Gewaltenteilung/-kontrolle gemessenen - Defizite beseitigt, wie auch die rechtlichen Grundlagen für ein Instrumentarium zur politischen Umsetzung geschaffen werden. Ausgehend von den Problemen der Verfassung von 1967 bzw. ihrer politischen Realität, werden im folgenden Lösungsansätze und Regelungen der Verfassung von 1992 skizziert, sowie die Hauptschwierigkeiten und Probleme, die noch bestehen bzw. jetzt auftreten.

\section{Menschenrechte, Grundrechte und Grundfreiheiten}

Die Verletzung von Menschenrechten und die Einschränkung von Grundrechten, insbesondere der politischen Mitwirkungsrechte, gehörten zur politischen Realität unter der Verfassung von 1967 wie auch der vorangegangenen. Das Problem war weniger mangelnde Festschreibung - die Menschenrechte, von der Abschaffung der Folter bis hin zur Meinungsund Pressefreiheit sind als Grundrechte in der Verfassung von 1967 enthalten - sondern vielmehr die häufige Verletzung durch staatliche Organe (z.B. Mißhandlungen und Folter in Untersuchungshaft ${ }^{26}$, Vorgehen von Polizei und Militär, Zwangsschließung von Presseund Rundfunkeinrichtungen, etc.). Die Aushöhlung der in der Verfassung statuierten Rechte wurde durch allgemein gehaltene Vorbehalte einzelner Grundrechte (z.B. Art. 71; 72; 118) erleichtert; diese Vorbehalte ließen sich durch weite Interpretation zugunsten des Vorrangs der Sicherung der bestehenden Ordnung zu gesetzlichen - den Wesensgehalt der verschiedenen Grundrechte antastenden - Einschränkungen ${ }^{27}$ mißbrauchen, die dann als Rechtsgrundlage und Vorwand für das Vorgehen gegen politische Gegner dienen konnten. Im Weiteren wurde der Exekutive die Möglichkeit zur Außerkraftsetzung bzw. Einschränkung von Grundrechten verfassungsrechtlich durch die Ausnahmezustandsregelung ermög-

Schon in den Debatten der verfassunggebenden Versammlung 1967 über die Verfahrensanforderungen bei der Verhaftung von Personen, wurde mit erstaunlicher Offenheit über die Praxis der Verhörungsmethoden mit Elektro-Schocks gesprochen, und von einigen Abgeordneten zusätzliche Schutzvorschriften gefordert - wofür sich jedoch keine Mehrheit fand. Vgl. die Diskussion um Art. 59 der verfassunggebenden Versammlung von 1967, a.a.O., Tomo II, Asunción 1974 , S. 337-376.

27

Als zwei Hauptstücke repressiver Gesetzgebung, gerichtet gegen Weltanschauungsfreiheit und Meinungsäußerungsfreiheit, können die Gesetze Ley No. 294 "De Defensa de la Democracia" (17.10.1955), sowie Ley No. 209 "De Defensa de la Paz Publica y Libertad de personas" (18.09.1970), genannt werden. Beide wurden im August 1989 widerrufen. 
licht (Art. 79; 181). Abgesehen davon, daß die Corte Suprema nicht die Kompetenz hatte, Gesetze im Wege der abstrakten Normenkontrolle für verfassungswidrig und allgemein unanwendbar zu erklären (die sie jetzt auch noch nicht hat), trug die Tatsache, daß die Gerichtsbarkeit weitgehend von der Exekutive abhängig war, zu diesem Umgang mit den Grundrechten bei; Klagen gegen Grundrechtsverletzungen durch Exekutivorgane wurden von der Justiz als während des Ausnahmezustands nicht judiziabel zurückgewiesen.

Die Verfassung von 1992 wertete den Schutz des menschlichen Lebens und seiner Integrität. So wurde das Recht auf Leben als natürliches, vorstaatliches Recht anerkannt (Art. 4), die Menschenwürde als gundlegender Wert, auf den jedes Regieren sich begründet, im ersten Artikel verankert - aus Gründen, die mit denen für die Garantie der Menschenwürde im deutschen Grundgesetz vergleichbar sind - , und das Verbot von Folter als eigener Artikel mit besonderer Bezugnahme auf politische Motive gefaßt (Art. 5). In diesem Zusammenhang wurde im Rahmen eines umfassenden Kapitels "Freiheit" eine Reihe von Normen zum Schutz vor bzw. bei Zugriff des Staates eingefügt. Dazu gehören vor allem strengere Verfahrensanforderungen bei Straf verfahren und Freiheitsentzug (Art. 11; 12; 17; 19) und die Statuierung der Unverletzlichkeit des Rechts an Identifikationspapieren und sonstigen persönlichen Dokumenten (Art. 35; 36). Nichtsdestoweniger sind die Verhältnisse in den Gefängnissen - wenn in den letzten Jahren auch deutlich verbessert - in vielerlei Hinsicht nicht gerade "menschenwürdig". Der Prozentsatz an festgenommenen Personen, die in Haft sitzen, ohne verurteilt zu sein, liegt - je nach Gefängnis - bei bis zu $90 \% .^{28}$

Auch die individuellen Freiheitsrechte und politischen Mitwirkungsrechte wurden ausgebaut und - anders als 1967 - an den Anfang der Verfassung gestellt. Die Schutzbereiche der verschiedenen Freiheitsrechte wurden im allgemeinen erweitert, sowie die zulässigen Einschränkungen klarer gefaßt bzw. auf Vorbehalte, die sich aus der Verfassung ergeben, reduziert (z.B. Meinungsäußerungs- und Pressefreiheit, Art. 26; Versammlungs- und Demonstrationsfreiheit, Art. 32). Die Religionsfreiheit erfuhr eine Erweiterung um die Freiheit der Weltanschauung, bei Anerkennung des Gewissenseinwandes z.B. als Grund für die Wehrdienstverweigerung - dessen Anerkennungsverfahren zwischen Vertretern von Militär und Kirche heftig umstritten ist -, und Abschaffung des Römisch-Katholischen als Staatsreligion (Art. 24; 37). Einige spezielle Artikel z.B. zum Schutz der Massenmedien als soziales Kommunikationsmittel, über das Recht auf Informationen, über die Ausübung der Pressetätigkeit, etc. wurden neu eingefügt und sind wohl als Ausdruck des verfassunggeberischen Willens, gerade das Rechtsgut Pressefreiheit besonders zu schützen, zu sehen. lichen Besichtigung der Gefängnisse "Buen Partor" und "Panchito Lopez" im Rahmen der alljährlich erfolgenden Gefängnisvisite der Richter und Staatsanwälte am 30.03.1996 während eines Forschungsauf enthalts in Paraguay. 
Innerhalb des Kapitels "Arbeitsrechte" wurden das Recht auf Gewerkschaftsbildung, das Streik- und Aussperrungsrecht umfassender gewährt (Art. 96; 97; 98).

In Verbindung mit der Bezeichnung der Demokratie als "partizipativ" (Art. 1) erweiterte man die politischen Mitwirkungsmöglichkeiten um die Bürgerinitiative (Art. 123) und das Referendum (Art. 121; 122). Mit Wahlrecht und Wahlpflicht und dem Recht auf Parteienbildung sind diese in einem eigenen Kapitel "Politische Rechte und Pflichten" zusammengefaßt.

Im Zuge der Verfassunggebung waren auch bisher vernachlässigte gesellschaftliche und soziale Probleme zur Sprache gekommen, deren Einbeziehung in das Grundstatut der zukünftigen Gesellschaftsordnung von den jeweiligen Fürsprechern gefordert wurde. Ein Ergebnis dessen ist die Einfügung eines Kapitels zum Schutz der Eingeborenenbevölkerung (Art. 62-67). Die Anerkennung der diesen ethnischen Gruppen zugestandenen Rechte wird als Teil des in der Verfassung betonten Aspekts der "pluralistischen" Demokratie gesehen. Im Kontext der Anerkennung der Pluralität der Gesellschaft steht auch die Gleichstellung des Guaraní mit Castellano als Amtssprache. Zuvor war Guaraní zwar als Staatssprache neben Castellano anerkannt, nicht jedoch als Amtssprache (1967:Art. 5), obwohl über die Hälfte der Bevölkerung - im Rahmen des insgesamt stark vorhandenen Stadt-Land-Gegensatzes vor allem die Landbevölkerung - ausschließlich Guaraní spricht. Trotz der verfassungsrechtlichen Gleichstellung werden nach wie vor sehr wenige Gesetze in Guaraní veröffentlicht. ${ }^{29}$ Das Problem der Landverteilung, welches in Paraguay - wie in vielen anderen Ländern Südamerikas - immer wieder zu Besetzungen von Grund durch die campesinos sin tierra (Bauern ohne Land) führt, hat die Neufassung des Kapitels "Über die Landreform" (1967:128-132) bewirkt. Im Zusammenhang mit dem in der Verfassung von 1992 neu eingeführten Sozialstaatsprinzip (Art. 1 spricht von "estodo social de derecho") wurden zahlreiche Normen mit sozialem Gehalt in die Verfassung aufgenommen. So wurde beispielsweise das in seinen Grundzügen vorhandene System einer umfassenden gesetzlichen Sozialversicherung mit Versicherungszwang für Arbeiter in abhängem Arbeitnehmerverhältnis und deren Familien auf verfassungsrechtlicher Ebene bestätigt ${ }^{30}$ (Art. 95), und ein Recht auf eine würdige Wohnstätte (Art. 100) statuiert. Bei dieser, wie auch bei verschiedenen anderen Normen (z.B. Art. 7: Recht auf eine ökologisch ausgeglichene und gesundheitsverträgliche Umwelt), stellt sich die Frage, wie weit ihre Bindungswirkung geht und inwiefern sie einklagbar sind, wenn im selben Artikel einer Formulierung als Recht die

Dies liegt teilweise in der Natur der Guaraní-Sprache begründet, die als Altagssprache v.a. der Landbevölkerung mit entsprechendem Wortschatz zum Ausdruck abstrakter Rechtsbegriffe nicht geeignet ist, bzw. durch die Notwendigkeit von Umschreibungen die Übersetzung erschwert.

30

Nur 9,8\% der wirtschaftlich aktiven Bevölkerung sind vom sozialen Sicherungssystem, welches wichtige Arbeitssektoren, und damit weite Teile der Bevölkerung ausschließt, erfaßt; Ramiro Barboza, Constitución de la Républica del Paraguay 1992. Conventión Nacional Constituyente, Tomo I (2Bde.), Asunción 1993 (CIDSEP-AID), S. 507. 
Formulierung als Staatszielbestimmung folgt, mit einer mehr oder weniger weitgehenden Verpflichtung des Staates zur Schaffung von Bedingungen zur Gewährleistung des Rechts.

\section{Verhältnis zwischen Exekutive und Parlament}

Die Verfassung von 1967, wie auch schon die von 1940, räumte der Exekutive eine sehr starke Stellung gegenüber dem Parlament ein. Durch Einschränkung der Befugnisse der Exekutive und Erweiterung der Kompetenzen des Kongresses wird in der Verfassung von 1992 versucht, das Verhältnis zwischen den beiden Gewalten mit effektiver gegenseitiger Kontrolle auszugleichen. In dieser Absicht wurde die Parlamentsauflösungsbefugnis des Präsidenten abgeschafft, ebenso die Möglichkeit, außerhalb der regulären Sitzungsperiode des Parlaments Dekrete mit Gesetzeskraft zu erlassen. Das zuvor ausschließlich dem Präsidenten zustehende Einberufungsrecht des Kongresses während der Sitzungspause wurde dahingehend geändert, daß dem Kongreß ein Recht zur außerordentlichen Selbsteinberufung und Sitzungsverlängerung zugesprochen wird (Art. 184) - ein Recht, das ihm seit der 1870er Verfassung nicht mehr zukam. Das bis dahin uneingeschränkte Recht des Präsidenten zur Ausrufung des Ausnahmezustandes (1967: Art. 181) wurde an die Zustimmung des Parlaments gebunden, dem Parlament ein eigenes Recht zur Erklärung des Ausnahmezustands und zu seiner jederzeitigen Aufhebung gegeben (Art. 288). Im Gesetzgebungsverfahren wurde die Regelung, die eine automatische Sanktionierung von Gesetzesprojekten der Exekutive vorsah, wenn diese innerhalb der laufenden Sitzungsperiode nicht mehr behandelt worden sind (1967: Art. 156), abgeschafft. ${ }^{31}$ Auch die Möglichkeit der Wiederwahl eines Präsidenten wurde ausgeschlossen (Art. 229).

Zur Stärkung des Kongresses wurde zudem eingeführt: die Möglichkeit zur Bildung von Untersuchungsausschüssen mit Beweiserhebungsrecht (Art. 195); die Einführung des juicio politico (politisches Verfahren) auch gegen Präsident, Minister, und andere, - nicht wie bisher nur gegen Mitglieder des Corte Suprema (1967: Art. 151 Nr.3) - mit der möglichen Folge der Amtsenthebung bei 2/3 Mehrheit in Abgeordnetenhaus und Senat (Art. 225); ein Zitier- und Interpellationsrecht gegenüber Ministern und höheren Beamten (Art. 193) und im Falle, daß dem nicht oder ungenügend Folge geleistet wird und bei einem Mißtrauensvotums von 2/3, die Empfehlung zur Ministerentlassung (Art. 194).

In Verbindung mit dem Parlamentsauflösungsrecht und der Befugnis, Dekretgesetze in Zeiten zu erlassen, in denen der Kongreß nicht tagt - auch zwischen Auflösung und Neuwahlen! -, ergab sich die Möglichkeit, Druck auf die Parlamentsmitglieder auszuüben, um gegen deren Willen Gesetze durchzubringen. 
Eine der verfassungsrechtlichen Hauptschwierigkeiten sind derzeit Abgrenzungsfragen bei den Kompetenzen der Gewalten und Organe; diese werden wohl zunächst vornehmlich Gegenstand von Rechtsstreitigkeiten sein.

\section{Staat und Militär}

In engem Zusammenhang mit der Übermacht der Exekutive unter den Verfassungen von 1940 und 1967 steht ein in lateinamerikanischen Staaten, und in s.g. Entwicklungsländern allgemein, häufiges Problem der Regierungspraxis und Staatsorganisation, welches in Paraguay während der Regierungszeit Stroessners besondere Ausmaße angenommen hat: das Verhältnis zwischen Staat und Militär. Im Falle Paraguays wurde eine enge Verflechtung von Exekutive, Partei und Militär vor allem durch eine Art Pakt zwischen der Coloradopartei und dem Militär erreicht, in dem Sinne, daß z.B. der Eintritt in eine Militärakademie automatisch die Mitgliedschaft in der Coloradopartei mit sich brachte; hinzu kommt die Tatsache, daß der Präsident - gemäß der Verfassung von 1967 zulässigerweise - Militär im aktiven Dienst war und der Coloradopartei angehörte, sowie die Praxis, daß Aufgaben der inneren öffentlichen Sicherheit und Ordnung durch Militär wahrgenommen wurden.

Dem Problem, daß sich die Exekutive das Militär zur Erfüllung anderer Aufgaben als nur der Landesverteidigung dienstbar gemacht hat, und andererseits das Militär maßgeblich an den politischen Entscheidungen mitgewirkt hat, haben die Verfassunggeber von 1992 versucht, durch verschiedene Maßnahmen zur Entpolitisierung des Militärs beizutragen. So sind Militär- und Polizeimitglieder im aktiven Dienst von der Übernahme des Präsidentenoder Vizepräsidentenamtes wie auch parlamentarischer Ämter ausdrücklich ausgeschlossen (Art. 235 Nr.7; 197 Nr.7); auch ist ihnen die Mitgliedschaft in Parteien und politischen Bewegungen, sowie die Ausübung von politischen Aktivitäten jeglicher Art untersagt (Art. 173; 175). Gleichzeitig ist die Abtretung des dem Präsidenten zugeschriebenen Oberbefehls über die Streitkräfte ausgeschlossen (Art. 238 Nr.9), damit auf diese Weise nicht umgangen werden kann, daß die oberste Leitung in der Hand des Zivil-Präsidenten bleibt. Um die Behandlung von Gesetzen, die das Militär betreffen, durch das Parlament in der jeweiligen Gesamtheit der Kammern zu sichern, ist - wie beim Staatshaushaltsgesetz, internationalen Verträgen und anderen Gesetzen von gleichgeschätzter Bedeutung - die Delegierung an Kommissionen untersagt (Art. 215).

Im Rahmen der Bemühungen um eine rechtliche Einordnung des Militärs in und unter die drei klassischen Staatsgewalten wurde auch die bisherige Eigenständigkeit der Militärgerichtsbarkeit insofern aufgehoben, als gegen Entscheidungen der Militärgerichte Rechtsmittel bei der ordentlichen Gerichtsbarkeit eingelegt werden können, und damit der Rechtsweg bis zur Corte Suprema als oberste Revisionsinstanz eröffnet ist (Art. 174; 259 Nr.3). 
Trotz aller verfassungs- und einfachgesetzlichen Versuche, den politischen Einfluß des Militärs zu verringern, ist die reale Machtstellung dieses - in der politischen Geschichte Paraguays schon immer sehr bedeutenden - Sektors nach wie vor sehr groß. Daß in ihm vor allem neben den Auswirkungen der Wirtschaftskrise - noch ein ernstzunehmendes Hinderungspotential für den im Gang befindlichen Demokratisierungsprozeß liegt, haben Ereignisse im April des Jahres 1996 gezeigt, als General Lino César Oviedo sich seiner Absetzung als Chef des Heeres widersetzte und seine Ernennung zum Verteidigungsminister forderte. ${ }^{32}$ Diese "stärkste Bedrohung der Demokratie Paraguays seit dem Sturz des Diktators Stroessner" ist schließlich - wohl auf Druck seitens Opposition, Parlament und Kabinettsmitgliedern sowie aus dem Ausland, insbesondere den U.S.A. - überwunden worden. $^{33}$

\section{Unabhängigkeit der Justiz}

Um die durch die Regelungen der Verfassung von 1967 ermöglichte Unterordnung der Judikative unter die Exekutive aufzuheben und eine unabhängige, kompetentere und effektivere Gerichtsbarkeit aufzubauen, ist die Organisation der rechtsprechenden Gewalt grundsätzlich reformiert worden. Das Grundmodell einer - ursprünglich am U.S.-amerikanischen System orientierten, allerdings modifizierten - Corte Suprema als oberste Instanz des gesamten Justizapparates mit gewissen verfassungsgerichtlichen Kompetenzen, hat die neue Verfassung beibehalten. Es wurde jedoch eine Gliederung in drei Senate - bestehend aus je drei Richtern - vorgenommen, von denen ein Senat ausschließlich für Verfassungsrechtsstreitigkeiten zuständig ist (Art. 258); die Festlegung der anderen Senate auf Zivil- und Handelsrecht zum einen und Strafrecht zum anderen erfolgte innerhalb des 1995 verabschiedeten Organgesetzes des Obersten Gerichtshofs. ${ }^{34}$ Diese Umstrukturierung hat zur Folge, daß Entscheidungen nicht mehr nur im Plenum getroffen werden, sondern vor allem in den einzelnen Senaten.

Die Ernennung der Richter der Corte Suprema erfolgt nicht mehr durch die Exekutive (1967: Art. 195), sondern durch den Senat - dem vorher ein Zustimmungsrecht zukam

Neue Zürcher Zeitung, 24. April 1996, S. 3; Frankfurter Allgemeine Zeitung, 25. und 27. April, S. 2 bzw. 8; El País, 28. April 1996, S. 8.

Dies und der so gut wie zwischenfallslose Verlauf der Munizipalwahlen am 17. November 1996 werden als Zeichen der Konsolidierung gesehen. Richtungsweisend für den Wandel wird die Wahl insofern eingeschätzt, als im Ergebnis mit Martin Burt, ein Kandidat der stärksten Oppositionspartei, der Liberalen, zum Bürgermeister gewählt worden ist. Economist, 30. November 1996, S. 52.

Ley No. 609 "que organiza la Corte Suprema de Justicia", vom 23. Juni 1995. 
(1967: Art. $151 \mathrm{Nr} .2)^{35}$ - auf Vorschlag eines neu eingeführten, vor allem im langwierigen Prozeß der Organgesetzgebung heftig diskutierten Consejo de la Magistratura ("Rat für den Richterstand"), mit Zustimmung der Exekutive (Art. 264 Nr.1). Das Bestreben, den Einfluß der Exekutive auf die Besetzung der richterlichen Ämter gering zu halten, und die Mitwirkung von Fachleute zu stärken, ist auch in der Zusammensetzung dieses Richterwahlgremiums deutlich erkennbar: nur ein Mitglied des acht-köpfigen Rates ist von der Exekutive ernannt; von den weiteren wird jeweils eines von Senat, Abgeordnetenhaus und Oberstem Gerichtshof gewählt, sowie jeweils zwei in Vertetung der Anwälte und der Rechtsprof essoren (Art. 262).

Die Amtzeit der Richter des Obersten Gerichtshofs, die zuvor auf fünf Jahre beschränkt war, und mit der Amtsperiode des Präsidenten zusammenfiel (1967: Art. 196), wurde in eine lebenslange umgewandelt, mit einer Altersbegrenzung von 75 Jahren. Sie können nur durch das Parlament im Wege des juicio politico ihres Amtes enthoben werden. Alle anderen Richter werden durch den Obersten Gerichtshof ernannt, ebenfalls auf Vorschlag des Consejo de la Magistratura (Art. 264 Nr.1; 2). Sie werden jeweils für einen Zeitraum von fünf Jahren ernannt, wobei die Ernennung nach zweimaliger Bestätigung zu einer lebenslangen wird. Während ihrer Amtszeit sind sie unabsetzbar und dürfen - als Reaktion auf eine zuvor übliche Praxis - auch nicht mehr versetzt werden (Art. 252). Ihre Amtsenthebung kann durch Entscheidung eines Jurado de Enjuiciamento de Magistrados erfolgen (Art. 253). Zur Gewähr der finanziellen Unabhängigkeit der Rechtsprechung ist ein Mindestbudget festgelegt worden, das mindestens 3\% des Haushaltsvolumens der Zentralverwaltung betragen muß (Art. 249).

\section{Schutz der Grundrechte und der verfassungsmäßigen Ordnung}

Dem Erfordernis, den früheren Aushöhlungsmöglichkeiten vor allem der Grundrechte effektiv entgegenzuwirken, wurde durch eine Verbesserung und Erweiterung des rechtlichen Schutzinstrumentariums entsprochen. Die Rechtsschutzverfahren erfuhren folgende Neuregelungen: Das Hábeas-Corpus-Verfahren zum Schutz vor Freiheitsentzug (1967: Art. 78) wurde derart ausgedehnt, daß neben den Fällen des schon erfolgten Freiheitsentzugs auch drohender Freiheitsentzug umfaßt ist, sowie sonstige Fälle von in diesem Zusammenhang stehenden physischen oder psychischen Beeinträchtigungen; für das Verfahren ist die Möglichkeit der Betreibung von Amts wegen vorgesehen (Art. 133). Die Klage kann bei jedem erstinstanzlichen Richter, wie auch beim Obersten Gerichtshof (Art. 259 Nr.4) erhoben werden. Neu eingeführt wurde das Hábeas-Data-Verfahren, mit welchem die Einsicht-

Die Zustimmung des Senats zu den Richteremennungen Stroessners war insofern kein Problem, als durch die bis 1990 geltende Wahlrechtsregelung, nach der 2/3 der Sitze in jeder Kammer auf die stärkste Partei entfielen, die Colorado-Partei mindestens 2/3 der Senatssitze innehatte. 
nahme in die in öffentlich geführten Registern und Akten gespeicherten personenbezogenen Daten und gegebenenfalls ihre Berichtigung oder Vernichtung gefordert werden kann. Diese Neuerung steht in engem Zusammenhang mit den unter der Diktatur Stroessners in den Polizeiarchiven gesammelten Informationen. Das Amparo-Verfahren, ein sehr weitgehendes Rechtsschutzverfahren, das auch in der Verfassung von 1967 vorgesehen war (1967: Art. 77), kann von jeder Person, die sich "durch eine Handlung oder Unterlassung, seitens einer staatlichen Behörde oder einer Einzelperson, in verfassungs- oder einfachgesetzlichen Rechten schwerwiegend verletzt fühlt oder in unmittelbar bevorstehender Gefahr, es zu werden, und dem aus Dringlichkeit des Falls nicht auf ordentlichem Rechtsweg abgeholfen werden kann", beschritten werden (Art. 134).

Eine weitere wichtige Änderung zugunsten der Grundrechte ist die Abschaffung der Möglichkeit, Grundrechte und deren Garantie- bzw. Schutzverfahren während des Ausnahmezustandes außer Kraft zu setzen. Die Exekutive kann allerdings wie zuvor die Verhaftung von Personen und den Ortswechsel anordnen, sowie Versammlungen und Demonstrationen untersagen (1967: Art. 79). Im Unterschied zu früher müssen Verhaftungen aber per Dekret für den Einzelfall geschehen und mit sofortiger Benachrichtigung der Corte Suprema über Person und Aufenthaltsort des einzelnen (Art. 288). Die nach wie vor bestehende Einschränkungsmöglichkeit wird dadurch relativiert, daß eine Ausnahmezustandsregierung durch das Zustimmungserfordernis des Parlaments wohl nur noch selten der Fall sein wird.

Im Sinne der der Judikative zugewiesenen Stellung als "Wächter der Verfassung" (Art. 247) sind die Zuständigkeiten des Obersten Gerichtshofs erweitert worden ${ }^{36}$. Die Möglichkeit, Gesetze für verfassungswidrig zu erklären, die nach der neuen Verfassung dem Verfassungssenat unterfällt, umfaßt nun auch Dekrete der Exekutive. Zudem können rechtskräftige Gerichtsentscheidungen als verfassungswidrig erklärt werden (Art. $260 \mathrm{Nr}$.1,2). Problematisch und heftig umstritten ist, daß - wie in der Verfassung von 1967 - die Verfassungswidrigkeitserklärung von Normen keine "erga-omnes" Wirkung, sondern nur einen "inter partes"-Effekt hat, d.h. nur zur Unanwendbarkeit im konkreten Fall führt. Damit bleiben die für verfassungswidrig befundenen Normen in Kraft, d.h. es erfolgt damit auch kein neuer Gesetzgebungsauftrag an die Legislative. Die Norm kann nur über den Weg des Gesetzgebungsverfahrens aufgehoben werden. Die Gründe für diese Regelung liegen vor allem in der Geschichte: das mangelnde Vertrauen in die Justiz und die Angst, daß damit die Judikative über das Parlament gestellt werden würde - über das Organ, zu dem als direkt vom Volk gewählten das größte Vertrauen besteht. Im Zuge weitergehender Justizreformen scheint die Einführung der "erga omnes"-Wirkung im Falle, daß Normen für verfassungswidrig erklärt werden, dennoch nicht völlig ausgeschlossen.

Siehe Art. 259 der Verfassung, sowie das Organgesetz der Corte Suprema Ley 609/95, insbes. Art. 3 und 11-13. 
Zudem ist der Aufgaben- und Kompetenzbereich des Obersten Gerichtshofs dadurch erweitert worden, daß er Kompetenzstreitigkeiten zwischen der Zentralverwaltung und den Departments, und zwischen diesen und den Gemeinden, zu entscheiden hat (Art. 259 Nr.9). Die Einführung dieser Zuständigkeit ist im Zusammenhang mit dem in der Verfassung von 1992 gestärkten Prinzips des dezentralisierten Einheitsstaats von besonderer Bedeutung; die neu eingeführten Kompetenzen der Departamentos und Municipios sollen davor geschützt werden, daß die traditionell stark zentralistische Verwaltung sie an sich zieht. Ein Organstreitverfahren zur Entscheidung von Kompetenzstreitigkeiten zwischen obersten Organen der Zentralebene, z.B. zwischen Parlament bzw. Senat und Präsident, ist nicht vorgesehen.

Neben Erweiterungen der Zuständigkeit des Obersten Gerichtshofs sind verschiedene Staatsorgane und -institutionen mit Überwachungs- und Kontrollfunktionen neu eingeführt worden. Als Teil der Judikative - wenn auch in teilweise umstrittenem Verhältnis zur Corte Suprema - ist die Justicia Electoral, eine besondere Wahlgerichtsbarkeit geschaffen worden, an deren Spitze ein Tribunal Superior de Justicia Electoral steht. Ihr ist die Organisation und Überwachung der Wahlen unterstellt, um die in den letzten Jahrzehnten üblichen Wahlmanipulationen zu unterbinden und für möglichst zwischenfallfreie Wahlen zu sorgen. Daneben ist der Defensor del Pueblo oder Ombudsman als Beauftragter des Kongresses zur Unteruchung der vorgebrachten Beschwerden über Verletzungen von Menschenrechten oder anderen in der Verfassung verankerten Rechten als Staatsorgan eingeführt worden. Noch im März 1996 war dieses Amt nicht besetzt, da man keine Einigung über den Kandidaten erzielte. Neu ist auch die Contraloría General de la Republica, bestellt im Zusammenwirken der beiden Kammern, zur Kontrolle der staatlichen Wirtschafts- und Finanztätigkeit, speziell der Überprüfung der Haushaltsausgaben.

\section{Schlußbemerkung}

Die in der Verfassung von 1992 getroffenen Neuregelungen, insbesondere im Bereich der Staatsorganisation, markieren einen bedeutenden Schritt zu einem demokratischen Regierungssystem mit ausgeglichener Gewaltenteilung und gegenseitiger Kontrolle, wenn sie sicherlich auch verschiedene Mängel haben. Weil in der Verfassungsgeschichte Paraguays die Verfassungsrealität vom Verfassungsrecht weit abwich, wird sich der Umfang des tatsächlichen Wandels erst mit der weiteren politischen Entwicklung zeigen. Die Verfassung von 1992 greift in Vielem auf die im Wege der Verfassungstradition entstandenen Strukturen zurück und steht, ob sie will oder nicht, im Licht dieser politischen Tradition; andererseits versucht sie mit verfassungsrechtlichen Neuerungen, sich davon zu lösen. Die Frage, ob die Umsetzung der in der Verfassung gelegten Grundlagen und Ziele für eine zukünftige paraguayische Gesellschaft möglich ist, hängt zunächst davon ab, inwieweit die mit den Neuerungen einhergehenden Probleme gelöst werden können. 
Wie erheblich die zu bewältigenden Schwierigkeiten noch sind, zeigt die Realität der einfachrechtlichen Umsetzung: Allein die Flut von neuen Gesetzen stellt in sich ein großes Problem dar, insbesondere für die Justiz, für die die Normenfülle ganz besondere Schwierigkeiten bei Anwendung und Umsetzung schafft. Die mangelnde Systematik der vorhandenen Gesetze und Dekret-Gesetze, sowie die häufig auftauchende unpräzise Klausel, "alles, was diesen Bestimmungen widerspricht, ist ungültig", erschweren die Übersicht über das geltende Recht. Die Umsetzung neuer Gesetzesprojekte, welche im Zuge ausländischer Rechtsberatung neue Rechtsfiguren und Verfahren mit sich bringen, benötigt gewiß die Weiterbildung der Rechtsanwender (Anwälte, Richter, Staatsanwälte) - und Zeit. 
of multipartyism in 1990, the mostly christianized non-Moslems support President Biyas RDPC party and oppose the UNCP of the Peul Bello Bouba Maigari. Furthermore, the Kirdi constitute a very heterogeneous group in itself divided by numerous conflicts.

\section{Human Rights in Sub-Saharan Africa: Towards Complementary Enforcement of Social Justice}

\section{By Sufian Hemed Bukurura}

The past and present decades are said to epitomize the era of good governance and transparency. It is in that context that constitutionalism, human rights and democracy are contemporary subjects of discussion all over the world. These concepts mean a lot to the organization of societies in general and legal systems in particular.

The current economic, political and social set-up of Sub-Saharan Africa does not seem to ensure that constitutionalism, human rights and democracy are readily available for the enjoyment of all people. The paper is a modest attempt in marking some signposts towards the exploration of alternative enforcement mechanisms, considering, inter alia, public interest litigation, social action litigation and participatory human rights enforcement.

\section{The Constitition of Paraguay of 1992 and its Predecessors}

\section{By Anja Schoeller-Schletter}

After decades of authoritarian rule, brought to an end in 1989 by the overthrow of General Alfredo Stroessner who dominated the country's politics since 1954, Paraguay is experiencing a process of transition from military dictatorship to democracy. A new constitution, passed in 1992, laid the legal foundation for a democratic government. As a reaction to the deficits of the preceding constitutions of 1967 and 1940, executive power was considerably restricted, the legislature was strengthened and the judiciary was subjected to fundamental reforms in order to guarantee its independence. Regardless of all changes in the normative system, tradition in terms of political practices and social structure turns out to be an impediment to the process of democratization.

The survey focuses on the solutions of the constitution of 1992 for the normative and political problems of the constitution of 1967: the extension of human rights protection; the abolition of the presidential authority to dissolve the Congress and the control of Congress 
over the declaration of the state of emergency; the incompatibility of military service and political mandate; the nomination of the judges of the Supreme Court by a newly created Consejo de la Magistratura and its appointment by the Senate - not by the president. The outline illustrates the Christian, hierarchical and patriarchal tradition of government in Paraguay as well as the recent changes and the difficulties still remaining. 